\title{
Responsibility toward affordable healthcare: disposable versus reusable methods for pelvic floor repair
}

\author{
Ivilina Pandeva' \\ Helen Johnson ${ }^{2}$ \\ Mark Slack' \\ Ashish Pradhan' \\ 'Department of Urogynecology, \\ Addenbrooke's Hospital, Cambridge, \\ UK; ${ }^{2}$ Department of Obstetrics and \\ Gynecology, Hinchingbrooke Hospital, \\ Huntingdon, UK
}

This article was published in the following Dove Press journal: International Journal of Women's Health

Background: The adverse publicity surrounding the use of mesh for correction of pelvic organ prolapse has driven a renewed interest in native tissue repair. Established techniques used reusable instruments, while recent innovations have generally involved disposable equipment. Here, we compare outcomes between the two techniques used for sacrospinous ligament fixation for the correction of apical prolapse: Miya Hook (reusable) and Capio ${ }^{\circledR}$ (single-use) suturing devices. Methods: A prospective cohort study of women undergoing vaginal sacrospinous colpopexy or hysteropexy was undertaken. The patients were assessed preoperatively and 1 year postoperatively. The primary outcomes were improvement in vaginal scores and patient-reported absence of a vaginal bulge. Secondary outcomes included impact on sexual function, quality of life, perioperative complications and apical recurrence. The cost of instruments, operative times and length of stay were analyzed.

Results: In total, 133 women with advanced prolapse in the apical compartment underwent colpopexy or hysteropexy (63 using the reusable Miya Hook and 40 with the disposable Capio device). Mean follow-up was 16.9 months (SD 7.8) and 14 months (SD 3.1), respectively. There were no significant differences in baseline characteristics or preoperative Pelvic Organ Prolapse Quantification staging between the two cohorts. Significant improvements in vaginal scores, sexual function and quality of life (International Consultation on Incontinence Questionnaire - Vaginal Symptoms) were observed in both groups postoperatively $(P<0.01)$. Both groups had low apical recurrence in the medium term $(<3 \%)$. There were no significant differences in operative times or length of stay. The use of disposable equipment was associated with higher cost.

Conclusion: Sacrospinous fixation using either the Miya Hook or the Capio device is equally effective for the management of apical prolapse. In the absence of clinical benefit, the choice of instrument should reflect the cost in a financially constrained health-sector environment.

Keywords: apical prolapse, native tissue repair, sacrospinous fixation, disposable instruments, reusable instruments

\section{Introduction}

Surgical techniques for the management of pelvic organ prolapse have advanced significantly in the past few decades, with increasing numbers of minimally invasive procedures being performed. Abdominal or laparoscopic sacrocolpopexy and sacrohysteropexy utilizing mesh augmentation were considered the most effective methods for correction of apical prolapse and are associated with a low risk of complication and a good long-term success rate. ${ }^{1}$ Because of the adverse publicity surrounding
Correspondence: Ivilina Pandeva Addenbrooke's Hospital, Hills Road, Cambridge CB2 0QQ, UK

Tel $+44 I 223586740$

Fax +44I22358659I

Email ivilina.pandeva@gmail.com 
the use of mesh, there has been renewed interest in native tissue repair, with patients increasingly choosing non-mesh alternatives such as sacrospinous fixation.

A range of procedures utilizing native tissues have been described for the correction of apical prolapse. ${ }^{2}$ Vaginal sacrospinous ligament fixation remains one of the most widely reported techniques. ${ }^{3}$ It was first described in 1958 by Sederl and involves suspension of the vaginal apex (cervix or vault) from the sacrospinous ligament (unilaterally or bilaterally), typically via the extraperitoneal approach and with the use of either delayed absorbable or permanent suture material. ${ }^{4}$ A variety of devices have been described to facilitate suture placement through the sacrospinous ligament. These include the Deschamps needle, the Miya Hook, the Capio ${ }^{\circledR}$ suturing device, the Veroniks ligature carrier and new reusable devices such as the SeraPro ${ }^{\circledR}$. None has been found to be superior to the others, but they differ in their technique for use, safety and cost profile. ${ }^{5-7}$

The attachment of the sutures to the sacrospinous ligament is achieved either by direct visualization during placement or using a "blind" approach relying on palpation during placement of the sutures. The latter requires less dissection and possibly carries a lower risk of soft tissue and vascular injury. ${ }^{8}$ The Miya Hook requires the use of long retractors to expose the tip of the needle to facilitate suture retrieval. This again raises the risk of soft tissue injury and may negate the advantages of the blind approach. Alternatively, the size of the bite taken in the ligament with the Capio device may be smaller than with the Miya Hook, potentially compromising the attachment and with it the long-term success of the procedure.

Direct comparative studies of the different techniques and devices available are sparse. In light of ever-increasing health costs and environmental concerns, we believe that it is important to compare reusable (Miya Hook) and disposable (Capio suturing device) instruments, to ensure that they are equally efficacious and carry no higher risks.

In this study, we compare the Miya Hook and the Capio suturing devices for placement of sutures through the sacrospinous ligament at the time of surgery.

\section{Materials and methods}

This prospective cohort study compared women undergoing vaginal sacrospinous colpopexy or hysteropexy for the treatment of apical prolapse at a specialist urogynecology unit between August 2014 and August 2015. Women were allocated sequentially: the first consecutive 63 patients underwent sacrospinous colpopexy or hysteropexy using the Miya Hook and the next 40 underwent the procedure with the use of the Capio suturing device.
Women were assessed preoperatively in a dedicated urogynecology clinic. The stage of pelvic organ prolapse was objectively assessed in the left lateral position with maximum Valsalva and after bladder emptying with the help of a Sims' speculum using the International Continence Society Pelvic Organ Prolapse Quantification (POP-Q) system. ${ }^{9}$

Subjective assessments of prolapse symptoms and their impact on overall quality of life (QoL) and sexual function were evaluated using the validated International Consultation on Incontinence Questionnaire - Vaginal Symptoms (ICIQ-VS) preoperatively and 12 months postoperatively. ${ }^{10}$

The primary outcomes were the patient-reported success rates and improvement in vaginal scores assessed with the use of the validated ICIQ-VS.

Secondary outcomes included impact on sexual function, QoL, perioperative complications and apical recurrence.

Operative times, length of stay and cost were recorded.

\section{Surgical procedure}

Sacrospinous fixation was performed under general anesthesia by one of the two consultant urogynecologists trained and competent in both techniques. All patients gave written informed consent prior to the procedure. Prophylactic coamoxiclav (or vancomycin and metronidazole if the patient was allergic to penicillin) were given at induction. Patients were positioned in the lithotomy position to allow transvaginal access. Access to the sacrospinous ligament, in both groups, was via the posterior compartment approach. The posterior vaginal wall was opened vertically in the midline, followed by sharp dissection to the rectovaginal fascia and further blunt dissection into the pararectal space on the right side of the patient. Landmarks of the region were identified by initial palpation and included the ischial spine and the sacrospinous ligament. The peritoneum overlying the ligament was pierced with the help of McIndoe scissors, allowing further dissection and skeletalization of the sacrospinous ligament. This helped to identify its margins and the fibrous anterior surface through which the ligament is penetrated, as described by Miyazaki. ${ }^{11}$

In the Miya Hook approach, two 0.0 polydioxanone sutures (PDS; Ethicon, Somerville, NJ, USA) were loaded on to the Hook simultaneously and inserted into the ligament unilaterally $2 \mathrm{~cm}$ medial to the ischial spine, taking care to keep the needle tip in the body of the ligament. This reduces the risk of vascular injury to the vessels situated posteriorly to the ligament. After placement of the sutures, Breisky-Navratil retractors were used for visualization of the Miya Hook tip and sutures as they exited the ligament. The sutures were then retrieved under direct vision using 
a nerve hook. During the use of the Capio suturing device (Capio, Boston Scientific Corp., Natick, MA, USA), identical dissection was undertaken to the right pararectal space unilaterally to allow insertion of two Capio device-specific monofilament polypropylene sutures sequentially. There was no need for the placement of retractors or the use of a retrieval device. ${ }^{12}$

The free ends of the sutures were secured to either the vaginal vault (in the absence of a uterus) or the posterior aspect of the cervix in the cases of a sacrospinous hysteropexy. Concomitant anterior and/or posterior vaginal wall prolapse was treated at the same time by anterior and/or posterior colporrhaphy. Some women required a vaginal hysterectomy, which was performed before the sacrospinous ligament fixation. At the end of the procedure, a vaginal pack and urinary catheter were inserted. These were removed on the following morning.

In this study, the principal surgical steps followed in performing the procedure were those described by Carey and Slack. ${ }^{8,13}$

\section{Statistical analysis}

The outcomes between the two groups were analyzed using independent Student's $t$-test for Gaussian data and Mann-Whitney test for nonparametric data using SPSS version 20.0 for Mac.

\section{Ethical approval}

Ethical approval for the project was granted by the Regional Ethics NRES Committee East Midlands, Derby (reference: 14/EM/0197). The study was conducted in accordance with the Declaration of Helsinki.

\section{Results}

During the study, 103 women with advanced prolapse in the apical compartment underwent colpopexy or hysteropexy (63 using the Miya Hook and 40 with the Capio suturing device).

Mean follow-up was 16.9 months (SD 7.8) in the Miya Hook group and 14 months (SD 3.1) in the Capio group.

There were no significant differences in age, parity and preoperative stage of prolapse in all compartments. Mean body mass index was higher in the Miya Hook than in the Capio group (Table 1).

The approach to the right sacrospinous ligament was via the posterior vaginal route and a concomitant posterior colporrhaphy was performed in $98 \%$ of patients in the Miya Hook group and in $95 \%$ in the Capio group. An anterior colporrhaphy was also performed in $83 \%$ and $78 \%$ of women and a vaginal hysterectomy in $39 \%$ and $23 \%$, respectively (Table 1). Overall, 33\% of women in the Miya Hook group and $20 \%$ in the Capio group had previously undergone prolapse repair (Table 1).

Table I Patient demographics and perioperative characteristics in the Miya Hook $(n=63)$ and Capio ( $n=40)$ groups

\begin{tabular}{|c|c|c|c|c|c|}
\hline \multirow[t]{2}{*}{ Demographics } & \multicolumn{2}{|l|}{ Miya group } & \multicolumn{2}{|l|}{ Capio group } & \multirow[t]{2}{*}{$P$-value } \\
\hline & Mean \pm SD & Range & Mean \pm SD & Range & \\
\hline Age (years) & $66.6 \pm 12.1$ & $37-87$ & $67 \pm 7$ & $5 I-83$ & 0.84 \\
\hline Parity (n) & $2 \pm 1.2$ & $0-6$ & $2.5 \pm 1.0$ & $0-6$ & 0.13 \\
\hline Body mass index $\left(\mathrm{kg} / \mathrm{m}^{2}\right)$ & $29.6 \pm 5.3$ & $21-44$ & $24.9 \pm 3.8$ & $20-31$ & 0.01 \\
\hline \multicolumn{6}{|l|}{ POP-Q } \\
\hline $\mathrm{Ba}$ & $2.0 \pm 1.1$ & $1-4$ & $2.5 \pm 0.8$ & $0-4$ & 0.2 \\
\hline C & $2.5 \pm 0.7$ & $1-4$ & $2.6 \pm 0.9$ & $1-4$ & 0.54 \\
\hline Bp & $2.5 \pm 0.6$ & $1-3$ & $2.8 \pm 0.5$ & $1-3$ & 0.2 \\
\hline Previous surgery & n (\%) & & n (\%) & & \\
\hline $\mathrm{TAH}$ & $14(22)$ & & $8(20)$ & & 0.98 \\
\hline $\mathrm{VH}$ & $16(25)$ & & $6(15)$ & & 0.87 \\
\hline PFR & $18(29)$ & & $8(20)$ & & 0.9 \\
\hline POP surgery & $21(33)$ & & $8(20)$ & & 0.85 \\
\hline Laparotomy & $3(5)$ & & I (3) & & 0.94 \\
\hline Concomitant procedure & n (\%) & & n (\%) & & \\
\hline$\overline{A R}$ & $5 I(83)$ & & 31 (78) & & 0.97 \\
\hline PR & $62(98)$ & & $38(95)$ & & 0.98 \\
\hline $\mathrm{VH}$ & $15(39)$ & & $9(23)$ & & 0.84 \\
\hline Other ${ }^{a}$ & $5(8)$ & & I (3) & & 0.88 \\
\hline
\end{tabular}

Note: a ${ }^{\circ}$ ther includes enterocele repair, trachelectomy, laparoscopic adhesiolysis and perineal tag repair.

Abbreviations: POP-Q, Pelvic Organ Prolapse Quantification; Ba, point B anterior; C, cervix; Bp, point B posterior; TAH, total abdominal hysterectomy; VH, vaginal hysterectomy; PFR, pelvic floor repair; POP, pelvic organ prolapse; AR, anterior repair; PR, posterior repair. 
Table 2 Preoperative and postoperative ICIQ scores in the Miya Hook and Capio groups (mean \pm SD)

\begin{tabular}{l|l|l|l|l|l|l}
\hline \multirow{2}{*}{ Scale } & \multicolumn{2}{|l}{ Miya group } & \multicolumn{3}{l}{ Capio group } \\
\cline { 2 - 7 } & Preop & Postop & P-value & Preop & Postop & P-value \\
\hline ICIQ-VS & $19.5 \pm 9$ & $4.9 \pm 6$ & $<0.01$ & $22 \pm 10.3$ & $4 \pm 7$ & $<0.01$ \\
ICIQ-SM & $29.7 \pm 17$ & $8.8 \pm I I$ & $<0.01$ & $23.8 \pm 13.2$ & $5.3 \pm 8$ & $<0.01$ \\
QoL & $6.5 \pm 3$ & $1.4 \pm 2$ & $<0.01$ & $7.5 \pm 3.4$ & $1.6 \pm 3$ & $<0.01$ \\
\hline
\end{tabular}

Abbreviations: IClQ, International Consultation on Incontinence Questionnaire; Preop, preoperative; Postop, postoperative; ICIQ-VS, International Consultation on Incontinence Questionnaire - Vaginal Symptoms; ICIQ-SM, International Consultation on Incontinence Questionnaire - Sexual Matters; QoL, quality of life.

Significant improvements in vaginal scores, sexual function and QoL from baseline were noticed in both cohorts $(P<0.01)$ (Table 2). There were no significant differences in postoperative vaginal scores, sexual function or QoL between procedures (Table 3). Similarly, there were no differences in complication rates, return to theater or blood transfusion between the groups. No patients in either group were readmitted for postoperative complications.

The median operative time was 66.48 minutes (SD 22.7) in the Miya Hook group and 67.65 minutes in the Capio group (SD 26.2). There was no significant difference in length of stay: 1.9 days (SD 0.28) in the Miya Hook group and 1.8 (SD 0.39) in the Capio group.

In the follow-up period, surgical failure in the apical compartment was observed in 3\% (2/63) of patients in the Miya Hook group and in $2.5 \%(1 / 40)$ in the Capio group $(P=0.98)$. New site anterior compartment prolapse was observed in four patients (6\%) in the Miya Hook group and in two (5\%) in the Capio group $(P=0.98)$.

There were no statistically significant differences in postoperative ICIQ scores, reoperation rates or the incidence of apical recurrence between the groups (Table 3).

In our institution, the reusable Miya Hook ligature carrier set includes a Miya Hook, Miya notched speculum, Adson nerve hook and Breisky-Navratil retractor, with a single upfront cost of $£ 245$. This can be used multiple times. The cost of sutures for each case where the Miya Hook carrier is used is $£ 10$, without any additional instrument sterilization costs as the Miya Hook set is included as part of the vaginal surgery set. In our case series, the cost of sacrospinous fixation equipment in the Miya Hook group was £14 per case.
In comparison, the cost of the disposable Capio suturing device is $£ 193$ and $£ 63$ for the device-specific sutures, with a total price of $£ 256$ per case.

\section{Discussion}

In this prospective cohort study, we demonstrate that sacrospinous fixation with both the Miya Hook and Capio suturing devices is associated with significant improvement in vaginal scores, sexual function and QoL in the medium term. The technique of sacrospinous fixation deserves new scrutiny in the light of the drive for native tissue repair for pelvic organ prolapse owing to the controversy surrounding mesh. ${ }^{14}$

The sacrospinous fixation procedure has high success rates and low complication rates despite the complex anatomy of the area and proximity of neurovascular and visceral structures. The short-term cure rates have been reported to be as high as $84 \%-100 \% .{ }^{1,15}$ However, the data on long-term success and failure rates are complicated partly by the use of different outcome measures and definitions of failure. Barber and Maher ${ }^{1}$ looked at 18 different definitions of surgical success at 2 years as part of the Colpopexy and Urinary Reduction Efforts Trial, and concluded that as a result of different definitions the perception of treatment success varies widely (from $19.2 \%$ to $97.2 \%$ ). Subjective or patient-reported measures of cure strongly correlated with treatment success and were more accurate than any other definitions. Anatomical success alone (defined as prolapse proximal to the hymen) had the lowest treatment success $(19.2 \%-57.6 \%){ }^{16}$

In our study, the patient-reported outcome measures were assessed with the use of validated QoL questionnaires. They have been shown to have a high correlation with long-term

Table 3 Comparison of postoperative ICIQ scores, reoperation rate and apical failure between the Miya Hook and Capio groups (mean \pm SD)

\begin{tabular}{l|l|l|l|l|l}
\hline Type of device & ICIQ-VS & ICIQ-SM & QoL & Reoperation rate & Apical failure \\
\hline Miya Hook & $4.9 \pm 6$ & $8.8 \pm I I$ & $1.4 \pm 2$ & $9.5 \%(6 / 63)$ & $3 \%(2 / 63)$ \\
Capio & $4 \pm 7$ & $5.3 \pm 8$ & $1.6 \pm 3$ & $7.5 \%(3 / 40)$ & $2.5 \%(1 / 40)$ \\
P-value & 0.7 & 0.4 & 0.3 & 0.95 & 0.98 \\
\hline
\end{tabular}

Abbreviations: ICIQ, International Consultation on Incontinence Questionnaire; ICIQ-VS, International Consultation on Incontinence Questionnaire - Vaginal Symptoms; ICIQ-SM, International Consultation on Incontinence Questionnaire - Sexual Matters; QoL, quality of life. 
treatment success and overall cure. We saw significant improvement in patient-reported vaginal symptoms, sexual function and QoL scores, with low surgical failure in the apical compartment of $<3 \%$. Similarly, a literature review by Lovatsis and Drutz showed successful results in $90 \%$ of cases, a low surgical failure rate $(3 \%)$ in the operated apical compartment, low perioperative complications and new site anterior wall prolapse of $5.8 \%{ }^{17}$ Pollak et $\mathrm{al}^{6}$ reported the complication rates of three different devices for sacrospinous fixation. They found that $5 \%$ of intraoperative and $17 \%$ of postoperative complications were related to the suture placement through the sacrospinous ligament. Although there were no statistically significant differences between the three groups, the study hypothesized that placement of the suture through the sacrospinous ligament under direct visualization may result in fewer intraoperative and postoperative complications. ${ }^{6}$ A review and cadaveric evaluation of six suture devices focusing on vascular safety concluded that anatomical variations and individual qualities of the sacrospinous ligament may affect their safety. ${ }^{5}$ They concluded that thinner and more compact devices such as the Capio or Caspari may be safer as they remain confined within the ligament and away from the neighboring neurovascular bundles.

However, we did not see a difference in perioperative complications between the Miya Hook and the Capio device groups. While this may be a phenomenon of powering, we believe that this reflects the fact that we routinely perform formal exposure of the sacrospinous ligament for both procedures. We feel that this technique ensures close attachment of the vaginal apex or cervix to the sacrospinous ligament, avoids bridging and encourages fibrosis for robust support. The main difference between the techniques relates to suture retrieval.

In our hands, as there is no significant increase in recurrence or complication rates, there would be a significant cost saving using the Miya Hook over the Capio. Cost analysis of operating theaters has shown that more than half of the budget is spent on equipment, with more than $80 \%$ of this being allocated to the purchase of disposable supplies. ${ }^{18}$ Sacrospinous fixation is one of the most widely performed prolapse procedures among UK surgeons for the management of primary and recurrent vault prolapse. ${ }^{19}$ On analysis of the British Society of Urogynaecologists database, we found that 574 sacrospinous procedures were performed between 2007 and 2010. ${ }^{20}$ Furthermore, between January 2000 and July 2018, there have been 5,006 sacrospinous fixation procedures using traditional reusable instruments $(4,451$ sacrospinous colpopexies and 555 sacrospinous hysteropexies) vs 5,190 sacrospinous fixations utilizing the Capio device (4,290 sacrospinous colpopexies and 900 sacrospinous hysteropexies). Considering the above data, the use of reusable instruments would have led to a cost saving of $£ 1,038,000$. While this may not seem to be a significant cost saving, it is only with an aggressive cost containment program in all aspects of our practice that we will bring down the overall cost of health.

Adler et $\mathrm{al}^{21}$ considered both the economic and environmental impact of single-use and reusable equipment in laparoscopic cholecystectomy, and found a 19 times higher cost of using disposable instruments. Furthermore, given the positive environmental impact of reusable equipment, they suggested that disposable instruments should be used only if there is a clear functional advantage. ${ }^{21}$

In our study, there were similar outcomes and improvement of symptoms with single-use and reusable equipment.

\section{Conclusion}

Sacrospinous fixation is a highly effective procedure irrespective of the instruments used. Techniques using the Miya Hook and Capio device for sacrospinous colpohysteropexy are equally effective in the treatment of apical prolapse and are associated with significant improvement in subjective outcomes, sexual function and QoL at 1 year. No difference was shown in time taken or complications experienced between the two techniques.

While we appreciate the occasional need in very selected cases for a lesser degree of dissection and a more refined, thinner instrument such as the Capio, in the absence of clinical benefit, the choice of instrument should reflect the cost in a financially constrained health-sector environment.

In the current era, further large multi-arm randomized controlled trials are warranted to inform us both on the efficacy and safety of various approaches for sacrospinous fixation and on their respective cost effectiveness.

\section{Author contributions}

Ivilina Pandeva: project concept and design; data acquisition; data analysis; manuscript drafting and revision; final approval of version for publishing; agreement to be accountable for all aspects of the work. Helen Johnson: project concept and design; data acquisition; manuscript revision; final approval of version for publishing; agreement to be accountable for all aspects of the work. Mark Slack: project concept and design; manuscript revision; final approval of version for publishing; agreement to be accountable for all aspects of the work. 
Ashish Pradhan: project concept and design; manuscript revision; final approval of version for publishing; agreement to be accountable for all aspects of the work.

\section{Disclosure}

The authors report no conflicts of interest in this work.

\section{References}

1. Barber MD, Maher C. Apical prolapse. Int Urogynecol J. 2013;24(11): $1815-1833$.

2. Maher C, Feiner B, Baessler K. Surgical management of pelvic organ prolapse in women. Cochrane Database Syst Rev. 2013;4:CD004014.

3. Barber MD, Brubaker L, Burgio KL, et al. Comparison of 2 transvaginal surgical approaches and perioperative behavioral therapy for apical vaginal prolapse: the OPTIMAL randomized trial. JAMA. 2014; 311(10):1023-1034.

4. Sederl J. Surgery in prolapse of a blind-end vagina. Geburtshilfe Frauenheilkd. 1958;18(6):824-828.

5. Manning JA, Arnold P. A review of six sacrospinous suture devices. Aust N Z J Obstet Gynaecol. 2014;54(6):558-563.

6. Pollak J, Takacs P, Medina C. Complications of three sacrospinous ligament fixation techniques. Int J Gynaecol Obstet. 2007;99(1):18-22.

7. Friedman T, Neuman M, Peled Y, Krissi H. A new reusable suturing device for vaginal sacrospinous fixation: feasibility and safety study. Eur J Obstet Gynecol Reprod Biol. 2015;193:23-26.

8. Randall CL, Nichols DH. Surgical treatment of vaginal inversion. Obstet Gynecol. 1971;38(3):327-332.

9. Bump RC, Mattiasson A, Bø K, et al. The standardization of terminology of female pelvic organ prolapse and pelvic floor dysfunction. Am J Obstet Gynecol. 1996;175(1):10-17.
10. Price N, Jackson SR, Avery K, Brookes ST, Abrams P. Development and psychometric evaluation of the ICIQ Vaginal Symptoms Questionnaire: the ICIQ-VS. BJOG. 2006;113(6):700-712.

11. Miyazaki FS. Miya Hook ligature carrier for sacrospinous ligament suspension. Obstet Gynecol. 1987;70(2):286-288.

12. Lind LR, Choe J, Bhatia NN. An in-line suturing device to simplify sacrospinous vaginal vault suspension. Obstet Gynecol. 1997;89(1):129-132.

13. Carey MP, Slack MC. Transvaginal sacrospinous colpopexy for vault and marked uterovaginal prolapse. Br J Obstet Gynaecol. 1994;101(6): 536-540.

14. NHS England. Mesh Oversight Group Report; July 2017. Available from: https://www.england.nhs.uk/wp-content/uploads/2017/07/meshoversight-group-report.pdf. Accessed October 10, 2018.

15. Sze EH, Karram MM. Transvaginal repair of vault prolapse: a review. Obstet Gynecol. 1997;89(3):466-475.

16. Barber MD, Brubaker L, Nygaard I, et al. Defining success after surgery for pelvic organ prolapse. Obstet Gynecol. 2009;114(3):600-609.

17. Lovatsis D, Drutz HP. Safety and efficacy of sacrospinous vault suspension. Int Urogynecol J Pelvic Floor Dysfunct. 2002;13(5):308-313.

18. Traverso LW, Hargrave K. A prospective cost analysis of laparoscopic cholecystectomy. Am J Surg. 1995;169(5):503-506.

19. Jha S, Cutner A, Moran P. The UK National Prolapse Survey: 10 years on. Int Urogynecol J. 2018;29(6):795-801.

20. Price N, Jackson SR, Foon R, Moran P. A comparison of vaginal sacrospinous fixation and abdominal sacrocolpopexy for vaginal vault prolapse repair in the UK: an analysis of the British Society of Urogynaecologists' (BSUG) database. International Continence Society; 2011. Available from: https://www.ics.org/Abstracts/Publish/105/000669.pdf. Accessed October 10, 2018.

21. Adler S, Scherrer M, Rückauer KD, Daschner FD. Comparison of economic and environmental impacts between disposable and reusable instruments used for laparoscopic cholecystectomy. Surg Endosc. 2005; 19(2):268-272.
International Journal of Women's Health

\section{Publish your work in this journal}

The International Journal of Women's Health is an international, peerreviewed open-access journal publishing original research, reports, editorials, reviews and commentaries on all aspects of women's healthcare including gynecology, obstetrics, and breast cancer. The manuscript management system is completely online and includes

\section{Dovepress}

a very quick and fair peer-review system, which is all easy to use. Visit http://www.dovepress.com/testimonials.php to read real quotes from published authors. 